\title{
Le rôle du faire-valoir indirect dans le renouvellement générationnel des agriculteurs irrigants en Tunisie
}

\author{
Hichem Amichi ${ }^{1, *}$, Jean-Yves Jamin ${ }^{2}$, Sylvie Morardet ${ }^{1}$, Inès Gharbi ${ }^{2,3,4}$, Amal Azizi ${ }^{2}$, Fadwa Faidani ${ }^{1}$, \\ Malak Ghileb $^{1}$, Serge Marlet ${ }^{2}$ et Mohamed Elloumi ${ }^{4}$ \\ 1 IRSTEA, UMR G-EAU, 361 rue Jean-François Breton, 34196 Montpellier, France \\ 2 CIRAD, UMR G-Eau, 73 rue Jean-François Breton, 34398 Montpellier, France \\ 3 INAT, 43 avenue Charles Nicolle, 1082 Tunis, Tunisie \\ ${ }^{4}$ INRA, rue Hédi Karray, 2049 Ariana, Tunis, Tunisie
}

\begin{abstract}
Résumé - L'accès des jeunes agriculteurs aux ressources foncières est peu pris en compte dans les débats sur la durabilité des agricultures dans les pays du Sud. Les dispositifs de transfert foncier vers cette catégorie sociale, importante sur le plan démographique, restent rares et peu opérants. Nous montrons ici, à travers l'étude de trois situations irriguées en Tunisie, que des transferts de terres vers les jeunes existent mais qu'ils s'effectuent souvent de manière informelle, et plus à travers différentes formes de faire-valoir indirect (FVI) qu'à travers un transfert de propriété. Nous analysons le rôle joué par le FVI dans la flexibilité des systèmes fonciers locaux et nous discutons le caractère durable des dynamiques qui en découlent. Nous discutons enfin le défi majeur que représente pour les décideurs politiques en Tunisie et au Maghreb l'inscription de cette agriculture informelle dans une perspective durable. Approfondir les connaissances sur le rôle joué par le FVI dans la flexibilité des systèmes fonciers et agraires locaux peut fournir des pistes de réflexion pour mieux valoriser des ressources en terre et en eau très disputées en Tunisie, et ailleurs au Maghreb.
\end{abstract}

Mots clés : faire-valoir indirect / renouvellement générationnel / jeunes ruraux / agriculture irriguée / Tunisie

\begin{abstract}
The role of indirect farming in the generational renewal of farmers in irrigated agriculture in Tunisia. Access to land for the young generation of farmers is hardly taken into account in the debates concerning agriculture sustainability in Southern countries. In these countries, where the population is often very young, procedures for transfer of agricultural assets are frequently absent and generally ineffective when they exist. We show in this article, through the study of three irrigated situations in Tunisia, that measures to transfer lands to a young generation of farmers operate informally through indirect farming. We analyze the role of indirect farming in creating flexibility in local land use systems and discuss the sustainability of the resulting dynamics. We finally discuss the major challenge for policy makers in Tunisia and North Africa to include informal agriculture in policy making to provide more sustainable perspectives. Better knowledge about the role of indirect farming in providing flexibility to local land use and agrarian systems can provide ideas for a better use of land and water resources, which are contested in Tunisia and elsewhere in North Africa.
\end{abstract}

Keywords: indirect farming / generational renewal / rural youth / irrigated agriculture / Tunisia

\section{Introduction}

La durabilité de l'agriculture dépend, comme le disent Godard et Hubert (2002), de « sa capacité à continuer à produire dans le futur, en entretenant les ressources naturelles et le capital humain ». Si la préservation des ressources naturelles est au centre de toutes les attentions depuis le rapport de Brundtland et al. (1987), l'importance du facteur humain est

\footnotetext{
* Auteur de correspondance : h.amichi@yahoo.fr
}

moins souvent mise en avant, même si Landais (1998) souligne l'importance des liens intergénérationnels pour la transmission des exploitations agricoles aux jeunes.

En Europe de l'Ouest, confrontée depuis les années 1950 au vieillissement de la population agricole et à un fort exode rural, des dispositifs institutionnels ont été mis en place pour encourager le transfert des exploitations des agriculteurs âgés vers des jeunes. Ainsi, des primes à l'installation et un contrôle foncier ont-ils été instaurés en France, afin d'encourager la reprise des exploitations, y compris par des règles de location favorisant les jeunes (Boinon, 2011). Dans les pays du Sud, où 
la population jeune occupe une place importante au plan démographique (en 2013, les 15 à 25 ans représentaient $18 \%$ de la population des pays en développement ; OIT, 2013), les dispositifs pour favoriser le transfert des exploitations aux jeunes sont rares et souvent inopérants quand ils existent. Les transmissions de terres s'effectuent essentiellement dans un cadre intrafamilial, conduisant, dans un contexte de forte croissance démographique, d'urbanisation et de diversification des usages des terres, à un morcellement important (Jouve, 2001).

Ce morcellement atteint aujourd'hui des proportions qui menacent la viabilité économique d'un grand nombre d'exploitations au Maghreb. Plus de $50 \%$ des agriculteurs exploitent moins de 5 ha en Algérie et en Tunisie; plus de $70 \%$ au Maroc. Pourtant des dispositifs de transfert existent, notamment sur les terres contrôlées par les États. Les réformes agraires, en Algérie et au Maroc, prévoient ainsi que la transmission des droits fonciers octroyés par l'État ne bénéficie qu'à un seul successeur, afin d'éviter le morcellement. Malgré cela, plusieurs travaux récents font état de l'échec des dispositifs administratifs visant à éviter le morcellement (Dugué et al., 2014 ; Imache et al., 2010). Ils montrent l'existence de très forts processus d'individualisation foncière, aussi bien sur les terres privées que sur les terres collectives ou étatiques. Ils sont particulièrement importants en Algérie, sur les terres publiques, suite à la division informelle des exploitations agricoles collectives (EAC) qu'avait créées l'État algérien (Amichi et al., 2015).

Dans les trois pays du Maghreb, les blocages ont conduit les acteurs à multiplier les arrangements contractuels, souvent informels, qui assurent le rôle de " connecteurs » entre des acteurs différemment lotis en ressources productives (Amichi et al., 2015 ; Ammar Boudjellal et al., 2011). Ces arrangements pallient ainsi l'insuffisance d'offre de crédit étatique ou la faiblesse du marché d'achat-vente des terres. Leur rôle dans l'installation des jeunes exploitants agricoles reste cependant peu abordé. En particulier, le rôle joué par le faire-valoir indirect (FVI) dans les dynamiques agricoles locales en Tunisie est peu documenté et considéré par les statistiques officielles comme étant très marginal.

À travers l'étude de trois situations agricoles irriguées en Tunisie, nous analysons la diversité des modalités d'accès au foncier et leurs fonctions pour les différents acteurs, en particulier pour les jeunes qui s'installent. Nous discutons également la durabilité des systèmes irrigués et la flexibilité qu'apportent les arrangements contractuels, formels et informels.

\section{Terrains d'études et méthode}

\subsection{Trois territoires irrigués contrastés en Tunisie}

Les trois zones d'étude ont été choisies le long d'un transect qui va du Nord-Ouest au Centre de la Tunisie (Fig. 1). Cela permet d'avoir trois situations agricoles qui ont en commun l'irrigation, et donc l'intensification et le dynamisme agricole qu'elle permet, avec leurs corollaires en termes d'attractivité et de tensions foncières ; mais aussi des situations contrastées au plan du climat, du système foncier local et des systèmes de culture. Dans la vallée de la Medjerda, le climat est sub-humide et certaines années le risque d'inondations est supérieur à celui des sécheresses, alors que dans la plaine de Kairouan le climat semi-aride réduit fortement l'agriculture pluviale ; la situation est intermédiaire dans les collines de Siliana. Ce contraste climatique se répercute sur les disponibilités en eau de surface dans chacune de ces zones. Dans la plaine de la Medjerda, l'eau d'irrigation est fournie à la fois par l'Oued Medjerda, le plus important cours d'eau de Tunisie, et le barrage de Bou Heurtma sur un de ses affluents. Siliana bénéficie aussi d'un barrage, même si les disponibilités en eau varient plus selon les années. Enfin, à Kairouan, les ressources en eau de surface sont très limitées, et l'irrigation n'a pu se développer que par l'accès aux eaux souterraines par des forages collectifs d'abord, aménagés par les pouvoirs publics et gérés dans le cadre des groupements de développement agricole (GDA), puis individuels et réalisés souvent sans autorisation préalable de l'administration.

Les périmètres irrigués de Siliana et de la Medjerda se distinguent par le fait que, à côté des exploitations agricoles anciennes, avec des terres de statut privé, Melk, une partie des terres relève du domaine privé de l'Etat et sont concédées à des sociétés de mise en valeur et de développement agricole (SMVDA), ou à des attributaires individuels, « jeunes agriculteurs » à Siliana, et «techniciens » dans la Medjerda. Ces attributions de l'État sont faites par des baux de longue durée (respectivement 15 et 40 années), sans cession de la propriété de la terre ni garantie sur le futur, même si, d'après les agriculteurs, des promesses de transfert leur auraient été faites à l'origine.

Après discussion avec les institutions agricoles (ministère et commissariats régionaux de développement agricole [CRDA], centres de recherche) et une étude bibliographique, un territoire irrigué a été choisi dans chaque zone, comme représentatif, au plan qualitatif, des problèmes et des dynamiques de cette zone, et non comme image statistique fidèle de ses réalités, difficiles à apprécier en l'absence de statistiques agricoles fiables, exhaustives et actualisées. Il s'agit du périmètre public irrigué (PPI) du Brahmi (4765 ha irrigués) dans la plaine de la Medjerda (grandes cultures céréalières, élevage laitier et maraîchage), du PPI de Gaâfour (1728 ha irrigués) dans les collines de Siliana (polyculture et marâ̂chage), des localités de Sidi Ali Ben Salem et Chebika Ouest (environ 800 ha irrigués) dans la délégation de Chebika, pour la plaine de Kairouan (maraîchage irrigué).

\subsection{Une démarche d'enquête en deux phases}

Les données ont été collectées d'abord par des entretiens exploratoires, puis par des enquêtes détaillées.

\subsubsection{Entretiens exploratoires}

Les entretiens ont commencé par la visite des administrations locales afin d'avoir une vision générale des structures d'exploitation, des principales productions, des modes de faire-valoir, et des conditions d'accès à l'eau. Ils ont concerné les structures publiques locales : CRDA, cellules territoriales de vulgarisation (CTV), GDA, Agence foncière agricole (AFA) et Office des terres domaniales (OTD). Ils ont aussi inclus certains acteurs des filières comme les fournisseurs d'intrants et de matériel d'irrigation, qui ont souvent une vision des agriculteurs et de leurs modes de production, plus précise et plus actuelle que les administrations locales. Enfin, des rencontres ont eu lieu avec 


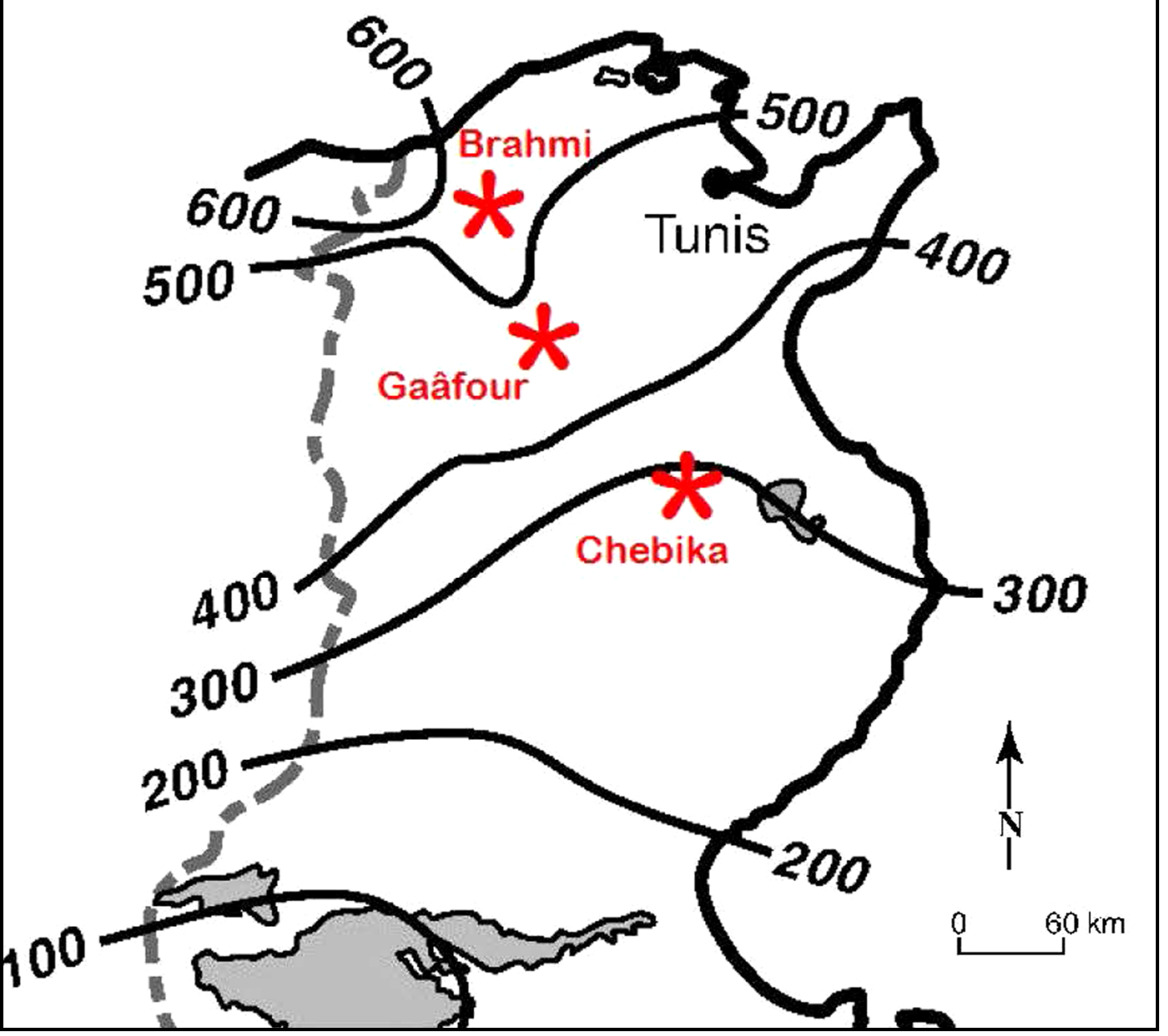

Fig. 1. Localisation des trois zones d'études en Tunisie (isohyètes d'après Benzarti, 2003).

Fig. 1. Location of the three study areas in Tunisia (isohyets according to Benzarti, 2003)

certains exploitants; elles ont été complétées par des observations directes des pratiques agricoles.

\subsubsection{Enquêtes détaillées auprès d'un échantillon d'irrigants}

Sur la base des données recueillies durant la phase exploratoire, un échantillonnage raisonné a été effectué pour prendre en compte la diversité des situations agricoles, sans pour autant viser une représentativité statistique. Nous avons étudié 110 exploitations agricoles (40 au Brahmi, 30 à Gaâfour et 40 à Chebika), de taille variée, ayant des systèmes de production différents (maraîchage, arboriculture, céréales) et accédant aux terres selon des modes de faire-valoir diversifiés (propriétaires, attributaires de terres domaniales, locataires et métayers). Chaque catégorie a fait l'objet d'enquêtes approfondies sur la base d'un questionnaire détaillé portant sur les pratiques foncières, les systèmes de production, les logiques d'acteurs (motivations des cédants et des preneurs de terres), la nature du lien et les rapports sociaux entre les contractants et les évolutions.

\section{Résultats}

Nous présentons ici des situations foncières dans lesquelles le FVI joue un rôle particulier face aux contraintes foncières, particulièrement pour les jeunes. Notre objectif n'est pas de présenter de manière exhaustive toutes les situations de FVI rencontrées, mais plutôt de mettre en avant quelques exemples de flexibilité que permet le FVI. Contrairement à ce qu'affirment les statistiques officielles, plus des deux tiers des exploitations enquêtées dans chacune des zones d'étude ont recours au FVI de manière ponctuelle ou fréquente (Tab. 1).

\section{1 À Chebika, le FVI au service d'une régulation intrafamiliale du foncier}

Dans cette localité, le lien familial est primordial dans les échanges fonciers : « Nous manquons de terres irrigables au sein de notre Douar, alors pourquoi les céder à un étranger quand elles sont inexploitées par l'un de nos proches? Nous mettons en avant les liens familiaux pour avoir accès de manière prioritaire 
Tableau 1. Importance des différents modes de faire-valoir au sein des exploitations agricoles enquêtées.

Table 1. Importance of the various modes of indirect farming within the surveyed farms.

\begin{tabular}{llll}
\hline & FVD (\%) & FVI (\%) & FVD + FVI (\%) \\
Chebika (40 exploitations) & 27,5 & 15 & 57,5 \\
Gaâfour (30 exploitations) & 35 & 45 & 20 \\
Brahmi (40 exploitations) & 30 & 32,5 & 37,5 \\
\hline
\end{tabular}

FVD : faire-valoir direct ; FVI : faire-valoir indirect.

aux terres qui se libèrent. Me concernant, j'exploite avec mes deux frères 2,5 ha irrigués que nous louons auprès d'un cousin, parti travailler en ville. L'impératif de rotation qu'exige le maraîchage nous contraint à chercher d'autres terres pour à la fois laisser nos terres en repos et conserver en même temps le même niveau de revenus. Vous imaginez donc qu'à la moindre possibilité de location ou de métayage offerte, nous sautons sur l'occasion. De plus, très peu de terres sont mises en vente, et quand elles le sont, elles sont hors de prix, en tous cas pour nous. » (Mostapha, 38 ans). Les motivations des preneurs de terres en FVI sont ainsi bien résumées : la majorité des agriculteurs familiaux dispose de très peu de terres. Or, ils ont besoin d'accroître leur superficie, à la fois pour des questions économiques (on rejoint ici la surface minimale viable mise en avant par le ministère de l'Agriculture et les organismes de crédit) et pour des questions agronomiques : la spécialisation, liée aux marchés et aux contraintes propres à chaque exploitation, conduit à des problèmes phytosanitaires ou, plus largement, de « fatigue des sols » qui imposent de faire des rotations ou de trouver de nouvelles terres ayant des précédents culturaux acceptables. Dans un contexte où les contraintes d'accès au foncier via l'achat-vente sont fortes (faible disponibilité des terres et prix jugé exorbitant par les agriculteurs quand les terres sont disponibles), c'est le FVI qui offre la possibilité à ces petits agriculteurs familiaux d'augmenter leurs superficies irriguées. La recherche de nouvelles terres proches de celles en propriété est également motivée par la nécessité d'avoir un accès à l'eau, obtenu, soit grâce au GDA (qui dessert un périmètre très précis), soit, de plus en plus fréquemment, grâce à un forage privé déjà existant. Louer des terres lointaines et sans accès à l'eau nécessiterait une forte mobilisation de capitaux pour établir un nouveau forage, ce que la plupart des agriculteurs familiaux interrogés ne sont pas en mesure de faire.

La cession des terres en FVI peut répondre aussi à des besoins de rotations qui s'imposent aux propriétaires. Ceux-ci, s'ils n'ont pas les moyens (moyens financiers ou main-d'œuvre familiale) de diversifier eux-mêmes leurs cultures, ont besoin de locataires ou de métayers pour le faire et pour intensifier, ce qui leur laisse ensuite, pour les céréales, des parcelles ayant reçu des engrais et ayant été sarclées.

Le FVI autorise la rencontre entre cédants et preneurs via une diversité d'arrangements contractuels, informels pour l'essentiel, qui permettent à chacune des parties de tirer profit de la mise en valeur des terres en fonction de leurs ressources financières, de leur âge et de leur force de travail, de l'accès à l'eau, etc. Trois types de contrats agraires sont utilisés à Chebika : la location, le métayage au cinquième et le métayage à la moitié (part reversée au propriétaire). Des variations internes à chaque configuration contractuelle existent, illustrant la forte flexibilité que recherchent les acteurs.

L'analyse des motivations des cédants et des preneurs montre que, lorsque les rapports de production sont favorables à l'une des parties, les propriétaires optent pour le contrat de métayage et les preneurs préfèrent le contrat de location. $\mathrm{Ce}$ constat concorde avec la littérature (Colin, 2003). Le choix final du type de contrat varie en fonction de trois critères : la disponibilité de la force de travail, la disponibilité des ressources financières et les conditions d'accès à l'eau des deux parties. Celui qui, cédant ou preneur, détient ces facteurs arrive souvent à imposer le contrat qu'il souhaite, de façon, d'une part, à réduire sa participation aux charges variables et, d'autre part, à minimiser les risques qui incombent à toute production agricole.

La disponibilité de la force de travail est souvent liée à l'âge des acteurs ; or, les preneurs sont dans leur majorité jeunes et les cédants sont en général d'un âge avancé. On est en présence d'une dynamique agricole de type agricultural ladder (Colin, 2003) dans laquelle les jeunes actifs, se trouvant en bas de l'échelle, commencent leur activité agricole avec comme principale ressource leur force de travail. Dans la version complète de la trajectoire socioprofessionnelle de type agricultural ladder, les jeunes actifs accumulent avec le temps savoir-faire, capital d'exploitation et capital financier, en passant par plusieurs modes de faire-valoir des terres (métayage, location) pour arriver à accéder aux terres en faire-valoir direct (FVD), en situation d'âge avancé. Arrivés à ce stade, où la force de travail devient rare généralement, ils cèdent à leurs tours les terres en FVI à des jeunes en début d'activité (Colin, 2003). Cette dynamique permet en particulier aux acteurs de dépasser les contraintes d'accès aux ressources en terres et peut être à l'origine d'un important processus d'intensification agricole (Ouendeno et al., 2015).

\subsection{Redistribution informelle des terres de l'État par le FVI dans le périmètre de Gaâfour}

À Gaâfour, les terres domaniales attribuées par l'État à des « jeunes agriculteurs » représentent $14 \%$ de la surface agricole utile (SAU) du PPI. Quatre-vingt-dix-huit agriculteurs ayant entre 25 et 40 ans à l'époque (fin des années 1980) ont été installés sur 288 ha, bénéficiant d'une superficie par exploitant variant de 2,5 à 3,5 ha irrigués. L'accès à la propriété foncière est exclu pour ces jeunes, mais l'attribution par l'État leur permet cependant d'être adhérents du GDA, de participer aux assemblées générales et de prétendre à un mandat électif. Les parcelles doivent être cultivées par chaque attributaire, leur sous-location à un tiers est explicitement interdite dans le bail passé avec l'État.

Quelques années après l'attribution des terres, on enregistre cependant des écarts aux règles édictées. Ainsi, certains attributaires en difficulté financière cèdent une partie ou la totalité de leurs terres à des tiers. Il existe donc un marché informel de FVI qui régule les disponibilités foncières entre bénéficiaires, d'une part, et entre bénéficiaires et autres types agriculteurs (non bénéficiaires), d'autre part. Le FVI assure ainsi plusieurs fonctions permettant de garantir la continuité de la production agricole sur les terres domaniales. Il se développe en réponse à plusieurs problèmes non considérés par 
la réforme agraire : le manque de financements pour l'agriculture irriguée, qui nécessite la mobilisation de capitaux importants alors que nombre d'exploitations sont trop petites pour avoir accès au crédit officiel (accessible seulement audessus d'un seuil de viabilité de 5 ha exploités légalement); ou la faible taille des exploitations, qui impose de chercher à s'agrandir pour constituer des superficies agricoles économiquement viables. Ces problèmes sont liés notamment à la rigidité des cadres de gestion qui sont trop souvent focalisés sur la redistribution des terres au plus grand nombre et qui ne tiennent pas compte de l'évolution des conditions socioéconomiques des bénéficiaires.

Céder en FVI une partie ou la totalité de ses terres pour une courte durée apparaît, pour certains jeunes agriculteurs, comme une stratégie de survie. Elle leur permet de constituer un capital ensuite réinvesti dans l'intensification sur ce qui leur reste de terres, ou dans d'autres secteurs économiques. Cela peut être accompagné, de manière temporaire, par une vente de leur force de travail en dehors de leurs exploitations afin d'augmenter leurs revenus. Cette stratégie est déployée pour faire face à un déficit de moyens propres, caractéristique de la situation des jeunes agriculteurs. Elle est utilisée également pour faire face à une offre de crédit, étatique ou privée, inexistante ou très peu opérationnelle car réservée aux exploitations ayant à la fois une surface minimale et des titres de propriété, laissant ainsi de côté tous les jeunes petits attributaires de lots, mais aussi tous ceux qui louent des terres.

Le FVI est aussi une réponse à la faible taille des lots attribués aux agriculteurs. En effet, $40 \%$ des attributaires ont des superficies de 2,5 ha et $60 \%$ ont des superficies de 3,5 ha. Ces agriculteurs nous ont fait part de la difficulté à rentabiliser leurs activités agricoles, notamment ceux qui cultivent 2,5 ha. L'impératif de faire des rotations pour éviter l'épuisement des sols exige la diversification des cultures, voire la pratique de la jachère. Cela réduit par conséquent la superficie qui peut être consacrée chaque année aux cultures maraîchères intensives. Les attributaires consacrent souvent la moitié de leurs terres aux cultures céréalières, dont le prix est garanti par l'État, et l'autre moitié au maraîchage. Deux possibilités se présentent alors aux attributaires pour assurer la survie de leur exploitation ou pour la développer. La première est de céder une partie ou la totalité de leurs terres à un autre agriculteur, mieux doté en capital, et d'exercer, quand l'occasion se présente, une activité saisonnière, agricole ou non. Cela correspond à une « stratégie de survie » et ne se matérialise souvent que par un contrat verbal de location de plusieurs années entre l'attributaire et un autre attributaire ou un agriculteur du secteur privé. La cession en FVI par des attributaires peut aussi s'expliquer dans certains cas par leur manque de technicité dans la pratique du maraîchage intensif, ce qui les contraint, pour mettre en valeur leurs terres, à faire appel à un associé quand c'est possible, sinon à un locataire. La seconde possibilité consiste à prendre des terres en FVI auprès d'un autre attributaire ou d'un exploitant privé, afin d'étendre les superficies en maraîchage. Cette seconde option correspond à une «stratégie de développement » qui nécessite des moyens et se matérialise souvent par un contrat de métayage à la moitié, permettant ainsi le partage des coûts avec un autre agriculteur. La mise en commun des moyens de production est encouragée à Gaâfour, par les opérateurs de la filière tomate de conserve, qui exigent une superficie minimale de 5 ha mitoyens pour passer des contrats avec les producteurs. Comme la superficie maximale par attributaire ne dépasse pas 3,5 ha, cultiver des tomates industrielles signifie forcément, pour ces attributaires, le recours au FVI via le métayage ou la location.

\subsection{Le FVI, facteur de déverrouillage de l'accès aux terres en indivision et aux terres domaniales dans le Brahmi}

Comme à Gaâfour, une partie du périmètre du Brahmi, appartenant au domaine privé de l'État, a été attribuée par celui-ci à des SMVDA et à des ex-agents du ministère de l'Agriculture (techniciens ou ingénieurs), appelés communément techniciens agricoles. Ici, ces techniciens ont reçu dix lots de 10 ha, avec un bail de 40 ans renouvelables. Comme à Gaâfour, on constate l'existence d'un marché de FVI embryonnaire, qui assure un rôle de régulation et de transfert de terres entre bénéficiaires et vers d'autres types d'agriculteurs. L'incapacité de certains attributaires à cultiver leurs terres, par manque de moyens financiers, a été évoquée comme principale motivation par les cédants. Nous retrouvons ici, de la même manière qu'à Gaâfour, deux stratégies utilisées par les attributaires : une stratégie de survie par la cession d'une partie ou de la totalité de leurs terres en FVI, avec exercice d'une activité en dehors de l'exploitation, et une stratégie de développement et d'agrandissement par la prise de terres en FVI auprès de leurs voisins bénéficiaires cédants, ou auprès de privés cédants.

Le cas du Brahmi permet aussi de discuter d'un aspect particulier du droit foncier tunisien (et maghrébin de manière générale), qui concerne le régime de transfert des terres Melk. L'application du régime successoral émanant du droit musulman entraîne une multiplication des ayants droit et implique inévitablement un morcellement des terres. Afin de l'éviter, le système de l'indivision est adopté par certains héritiers, notamment lorsque les ayants droit sont nombreux ou lorsqu'ils ne peuvent pas payer les droits de mutation. Mais ce choix de l'indivision peut aussi constituer un frein à la mise en valeur des terres, du fait des conflits entre ayants droit sur l'usage et la répartition des parcelles. Nos enquêtes montrent que ce blocage est récurrent sur les terres Melk irriguées du Brahmi. Cela peut aboutir, alors que l'on se situe dans un PPI où l'État a investi dans les infrastructures, à ce que les terres ne soient plus cultivées. Quand elles le sont, le statut foncier instable ne sécurise pas les investissements à long terme, interdisant par exemple l'arboriculture. Nos enquêtes montrent également que le recours de certains héritiers au FVI permet de déverrouiller la mise en valeur de terres Melk en indivision : la totalité de la propriété est cédée en location, par contrat écrit, à un preneur qui la cultive contre une rente foncière qui sera partagée de manière égale ou selon la règle du droit musulman entre tous les ayants droit. Le locataire des terres peut être un des ayants droit, ce qui peut permettre à un des héritiers de s'installer, même sans avoir la propriété des terres. Le loyer obtenu par la location des terres familiales peut aussi permettre à certains héritiers de louer à leur tour d'autres terres (Melk ou lots de techniciens).

\section{Discussion et conclusion}

L'analyse des conditions d'accès au foncier dans ces trois zones irriguées montre l'existence d'un marché de FVI 
dynamique, permettant une diversité d'arrangements contractuels entre cédants et preneurs. Cela illustre la théorie du changement institutionnel dans le champ foncier (Colin, 2004), selon laquelle le jeu combiné de la pression démographique, du développement des cultures irriguées destinées au marché et des changements dans les systèmes de culture, augmente la valeur de la terre et conduit à l'ouverture de l'éventail des droits de transfert et à leur monétarisation. Nous avons vu ici la flexibilité que le FVI peut conférer aux systèmes fonciers et agraires locaux, en ouvrant le champ des transactions foncières entre cédants et preneurs. Il permet ainsi une rencontre plus facile entre l'offre et la demande de terre, moins contraignante que les transferts par achat-vente, comme l'ont aussi rapporté Deininger et Feder (2001) et Colin (2003). Le FVI permet aussi des pratiques agricoles bénéfiques en termes de rotation des cultures, et par le partage des coûts qu'il autorise, le transfert de savoir-faire technique, concernant le maraîchage en particulier ; il contribue donc au dynamisme de l'agriculture irriguée.

Nous avons observé que le renouvellement générationnel opéré grâce au FVI est souvent provisoire. À Chebika, c'est un modèle de gestion temporaire de la petite taille des exploitations qui permet aux agriculteurs de type familial de s'agrandir et de respecter les rotations culturales exigées par leur système maraîcher intensif. À Gaâfour, il permet de dépasser la situation de blocage générée par le cadre rigide de répartition et de gestion des terres domaniales. La redistribution des terres entre les attributaires et les tiers reste cependant précaire du fait de son caractère informel. En l'absence d'un système de crédit efficient, le FVI offre aussi des possibilités de financement aux attributaires des terres domaniales, par la cession partielle et temporaire de leurs terres. Enfin, dans le Brahmi, le FVI permet de déverrouiller l'accès aux terres domaniales et aux terres Melk en offrant une alternative au système d'indivision qui bloque parfois la mise en valeur des terres. Dans les trois situations, l'accès des nouveaux exploitants aux terres, provisoire et parfois précaire, ne leur permet pas cependant de se projeter dans le futur, notamment par des investissements à long terme. Ceci conduit à relativiser le caractère durable des dynamiques agricoles qui en découlent. Il apparaît donc une inadéquation entre une agriculture qui se veut intensive en capital, mais qui peine à se pérenniser, et le développement d'arrangements informels qui donnent une flexibilité à cette agriculture, mais avec une portée limitée dans le temps.

Dans les trois localités étudiées, la tendance observée en termes de pratiques de FVI est une progression de la location au détriment du métayage. Cette tendance, confirmée par les dires des agriculteurs, témoigne d'une évolution des rapports de production plus favorable aux tenanciers. À Chebika, le métayage à la moitié perd ainsi de l'importance au profit du métayage au cinquième (part reversée au propriétaire), plus favorable au métayer. Dans les périmètres de Gaâfour et du Brahmi, le contrat de location est dominant dans les modes de FVI ; le métayage à la moitié constitue désormais l'exception. De la même manière, la disparition des anciennes formes de contrats agraires, largement favorables aux propriétaires terriens, illustre cette inversion de situation. L'ancien système Khammès (métayage au cinquième dans lequel les quatre cinquièmes du revenu net revenaient au propriétaire), qui traduisait la domination des propriétaires sur les tenanciers, a pratiquement disparu aujourd'hui. La part revenant au métayer était alors d'un cinquième de la récolte, alors qu'aujourd'hui il lui en revient les quatre cinquièmes, comme à Chebika. Cela illustre à la fois un renversement des rapports de force, qualifié de reverse tenancy dans la littérature (Colin, 2014) et le fait que, dans une agriculture irriguée intensive, maraîchère en particulier, la terre n'est plus qu'un intrant parmi d'autres, avec des coûts beaucoup plus importants pour l'eau, les semences, les engrais, les produits phytosanitaires et le travail. Cela illustre aussi le caractère évolutif de ces contrats qui leur confère une forte capacité d'adaptation aux nouvelles contraintes agricoles et assure ainsi leur continuité dans le temps. L'évolution des rapports de production en faveur des tenanciers est aussi observée dans les nouvelles dynamiques maraîchères du Sud algérien: Hamamouche et al. (2015) montrent que les mutations à l'œuvre dans les oasis de Biskra se traduisent notamment par l'ascension économique et sociale des jeunes descendants des Khammès qui accèdent aux terres grâce au FVI et à la réalisation de forages pour l'irrigation.

Cette inversion des rapports sociaux de production en faveur des tenanciers et la prédominance du mode de fairevaloir mixte et temporaire dans les trois terrains d'étude et dans d'autres territoires irrigués du Maghreb indiquent une situation transitoire et non stabilisée du foncier et des exploitations agricoles. Cette transition, quand elle s'opère dans un cadre informel, peut contenir des fragilités à la fois sur les plans socio-économique et environnemental. Certaines de ces conséquences sont déjà avérées et mentionnées dans des contextes similaires en Algérie et au Maroc. Sur les terres publiques décollectivisées en Algérie, un fort processus de différenciation des acteurs a été constaté, qui profite pour l'essentiel à une minorité d'agriculteurs privés en ascension économique (Amichi et al., 2015). Ce sont souvent les acteurs qui ont un « capital social » important qui arrivent à capter les crédits et subventions étatiques permettant leur agrandissement par le FVI. De la même manière au Maroc, on constate le développement de l'accès des investisseurs aux terres collectives suite à la réforme de la main levée permettant la vente de ces terres (Ameur et al., 2013). Les investisseurs y ont accédé aux terres d'abord via le FVI et ensuite par des achats, entraînant un processus de concentration foncière.

Des limites environnementales ont été également mentionnées du fait de l'impact possible de ces dynamiques informelles sur la dégradation de la structure des sols et leur salinisation dans certains territoires irrigués du Maghreb (Douaoui et Hartani, 2008). Les contrats de courte durée peuvent en effet pousser le preneur à un usage minier des sols. Contraint de rentabiliser le plus rapidement possible son investissement, le tenancier ayant un bail de courte durée pourrait avoir tendance à pratiquer plusieurs cycles de culture sans observer de rotation culturale, ou à ne pas apporter les amendements organiques nécessaires à la reproduction de la fertilité du sol.

Le défi majeur qui se présente aujourd'hui aux décideurs politiques est de savoir comment inscrire cette agriculture, dynamique mais largement informelle, et caractérisée par des fragilités socio-économiques et environnementales, dans une perspective durable. Approfondir les connaissances sur le rôle joué par le FVI dans la flexibilité des systèmes fonciers et agraires locaux peut fournir des pistes de réflexion pour mieux valoriser des ressources en terre et en eau très disputées, en Tunisie et ailleurs au Maghreb. 
Remerciements. Ces travaux ont été menés dans le cadre du réseau Sirma (Systèmes irrigués au Maghreb) et ont bénéficié $\mathrm{du}$ soutien des projets: DAIMA (Dynamiques agricoles irriguées au Maghreb, AIRD), AMETHYST (évolution conjointe des ressources et des usages de l'eau en méditerranée, ANR), PAP-AGIR (Programme d'actions-pilotes d'appui aux groupements d'irrigation, AFD) et Eau4Food (European Union and African Union cooperative research to increase food production in irrigated farming systems in Africa, UE).

\section{Références}

Ameur F, Hamamouche MF, Kuper M, Benouniche M. 2013. La domestication d'une innovation technique : introduction et diffusion du goutte-à-goutte dans deux douars au Maroc. Cah. Agric. 22 (4): 311-318. doi:10.1684/agr.2013.0644

Amichi H, Bouarfa S, Kuper M. 2015. Arrangements informels et types d'agricultures sur les terres publiques en Algérie : quels arbitrages ? Rev. Tiers Monde 221(1): 47-67. doi:10.3917/ rtm.221.0047

Ammar Boudjellal A, Bekkar Y, Kuper M, Errahj M, Hammani A, Hartani T. 2011. Analyse des arrangements informels pour l'accès à l'eau souterraine sur les périmètres irrigués de la Mitidja (Algérie) et du Tadla (Maroc). Cah. Agric. 20(1): 85-91. doi:10.1684/ agr.2010.0458

Benzarti Z. 2003. La pluviométrie en Tunisie : analyse des années très pluvieuses. In: Arnould P, Hotyat M, eds. Eau et environnement : Tunisie et milieux méditerranéens.Lyon: ENS Edition, 63-78.

Boinon J-P. 2011. Les politiques foncières agricoles en France depuis 1945. Econ. Stat. 444(1): 19-37.

Brundtland GH, Khalid M, Agnelli S, Al-Athel S, Chidzero B, Fadika L, et al. 1987. Our common future. Report of the World Commission on Environment and Development. New York: United Nations.

Colin J-P. 2003. Figures du métayage. Étude comparée des contrats agraires (Mexique). Paris (France): IRD éditions.
Colin J-P. 2004. Le marché du faire-valoir indirect dans un contexte africain. Éléments d'analyse. Econ. Rurale 282: 19-39.

Colin J-P. 2014. La concentration foncière par la tenure inversée (reverse tenancy). Etudes Rurales 194(2): 203-218. www.cairn. info/revue-etudes-rurales-2014-2-page-203.htm

Douaoui A, Hartani T. 2008. Impact de l'irrigation par les eaux souterraines sur la dégradation des sols de la plaine du Bas-Chéliff. In: Kuper M, Zaïri A, eds. Économies d'eau en systèmes irrigués au Maghreb. Actes du troisième atelier régional du projet Sirma, Nabeul, Tunisie, 4-7 juin 2007. Montpellier (France): Cirad. http://hal.cirad.fr/cirad-00259785.

Deininger K, Feder G. 2001. Land institutions and land markets. Handb. Agric. Econ. 1: 288-331.

Dugué P, Lejars C, Ameur F, Amichi F, Braiki H, Burte J, et al. 2014. Recompositions des agricultures familiales: une analyse comparative dans trois situations d'irrigation avec les eaux souterraines. Tiers Monde 220: 99-118.

Godard O, Hubert B. 2002. Le développement durable et la recherche scientifique à l'INRA. Rapport intermédiaire de mission. Paris (France): Inra éditions.

Hamamouche F, Lejars C, Kuper M. 2015. Déverrouillage de l'accès aux ressources et émancipation de jeunes agriculteurs des oasis de Sidi Okba dans le Sahara Algérien. Cah. Agric. 24(6): 412-419. doi:10.1684/agr.2015.0777

Imache A, Hartani T, Bouarfa, S, Kuper M. 2010. La Mitidja 20 ans après: réalités agricoles aux portes d'Alger. Alger (Algérie): Éditions Alfa.

Jouve A-M. 2001. Terres méditerranéennes: le morcellement, richesse ou danger? Paris (France): Karthala Editions.

Landais E. 1998. Agriculture durable: les fondements d'un nouveau contrat social. Courr. Environ. INRA 33: 5-22.

OIT. 2013. Tendances mondiales de l'emploi des jeunes 2013 : une génération menace. Genève: BIT. http://www.ilo.org/wcmsp5/ groups/public/—dgreports/—dcomm/documents/publication/ wcms_222657.pdf.

Ouendeno M-L, Daoudi A, Colin J-P. 2015. Les trajectoires professionnelles des jeunes dans la néo-agriculture saharienne (Biskra, Algérie) revisitées par la théorie de l'agricultural ladder. Cah. Agric. 24(6): 396-403. doi:10.1684/agr.2015.0793

Cite this article as: Amichi H, Jamin J-Y, Morardet S, Gharbi I, Azizi A, Faidani F, Ghileb M, Marlet S, Elloumi M. 2016. Le rôle du fairevaloir indirect dans le renouvellement générationnel des agriculteurs irrigants en Tunisie. Cah. Agric. 25: 35004. 